\title{
SPECTRAL ANALYSIS OF CERTAIN SPHERICALLY HOMOGENEOUS GRAPHS
}

\author{
JonATHAN BREUER AND MATTHiAs KELLER
}

Abstract. We study operators on rooted graphs with a certain spherical homogeneity. These graphs are called path commuting and allow for a decomposition of the adjacency matrix and the Laplacian into a direct sum of Jacobi matrices which reflect the structure of the graph. Thus, the spectral properties of the adjacency matrix and the Laplacian can be analyzed by means of the elaborated theory of Jacobi matrices. For some examples which include antitrees, we derive the decomposition explicitly and present a zoo of spectral behavior induced by the geometry of the graph. In particular, these examples show that spectral types are not at all stable under rough isometries.

Mathematics subject classification (2010): 47B39 (primary), 81Q10, 05C50, 39A70 (secondary).

Keywords and phrases: Graph Laplacian, antitrees, singular continuous spectrum, absolutely continuous spectrum, rough isometry, Jacobi matrices, decomposition, symmetries, Remling's theorem.

\section{REFERENCES}

[1] C. Allard, R. Froese, A Mourre estimate for a Schrödinger operator on a binary tree, Reviews in Mathematical Physics 12, 12 (2000), 1655-1667.

[2] J. BReUER, Singular continuous spectrum for the Laplacian on certain sparse trees, Commun. Math. Phys. 269 (2007), 851-857.

[3] J. BREuER, Singular continuous and dense point spectrum for sparse trees with finite dimensions, in "Probability and Mathematical Physics: A Volume in Honor of Stanislav Molchanov", (eds. D. Dawson, V. Jakšić and B. Vainberg), CRM Proc. and Lecture Notes 42 (2007), 65-84.

[4] J. BReuer AND R. L. Frank, Singular spectrum for radial trees, Rev. Math. Phys. 21, 7 (2009), $1-17$.

[5] R. Froese, D. Hasler AND W. SPITZER, Transfer matrices, hyperbolic geometry and absolutely continuous spectrum for some discrete Schrödinger operators on graphs, Journal of Functional Analysis 230 (2006), 184-221.

[6] R. Froese, D. HASLER AND W. SPITZER, Absolutely continuous spectrum for a random potential on a tree with strong transverse correlations and large weighted loops, Rev. Math. Phys. 21, 6 (2009), 709-733.

[7] S. GolÉnIA, $C^{*}$-algebras of anisotropic Schrödinger operators on trees, Ann. Henri Poincaré 5, 6 (2004), 1097-1115.

[8] S. GolÉNIA, Unboundedness of adjacency matrices of locally finite graphs, to appear in Letters in mathematical physics, Lett. Math. Phys. 93, 2 (2010), 127-140.

[9] V. GeORGESCU, S. GoLÉNIA, Isometries, Fock spaces and spectral analysis of Schrödinger operators on trees, Journal of Functional Analysis 227 (2005), 389-429.

[10] A. Grigor'yan, X. Huang, J. Masamune, On stochastic completeness of jump processes, Math. Z. 271, Issue 3-4, (2012) 1211-1239.

[11] X. HuAnG, On stochastic completeness of weighted graphs, Ph.D. Thesis, University of Bielefeld.

[12] M. KeLLER, Essential spectrum of the Laplacian on rapidly branching tessellations, Math. Ann. 346, 1 (2010), 51-66.

[13] M. Keller AND D. LenZ, Dirichlet forms and stochastic completeness of graphs and subgraphs, Journal für die reine und angewandte Mathematik (Crelles Journal) 2012, 666 (2012), 189-223. 
[14] M. Keller, D. LenZ And R. K. Wojciechowski, Volume Growth, Spectrum and Stochastic Completeness of Infinite Graphs, preprint 2011, arXiv:1105.0395v1, to appear in Math. Z.

[15] Y. Last And B. Simon, The essential spectrum of Jacobi, Schrödinger, and CMV operators, J. d'Analyse Math. 98 (2006), 183-220.

[16] V. MÜLLER, On the spectrum of an infinite graph, Linear Algebra Appl. 93 (1987), 187-189.

[17] M. MAntoiu, S. Richard ANd R. Tiedra DE Aldecoa, Spectral analysis for adjacency operators on graphs, Ann. Henri Poincaré 8 (2007), 1401-1423.

[18] K. NAimark AND M. SOlOMYAK, Eigenvalue estimates for the weighted Laplacian on metric trees, Proc. London Math. Soc. 80 (2000), 690-724.

[19] M. Reed And B. Simon, Methods of Modern Mathematical Physics, I: Functional Analysis, Academic Press, New York, 1972.

[20] C. Remling, The absolutely continuous spectrum of Jacobi matrices, Annals of Math. 174 (2011), $125-171$.

[21] M. Schmied, R. Sims, G. Teschl, On the absolutely continuous spectrum of Sturm-Liouville operators with applications to radial quantum trees, Oper. Matrices 2, 3 (2008), 417-434.

[22] B. Simon And G. Stolz, Operators with singular continuous spectrum, V: Sparse potentials, Proc. Amer. Math. Soc. 124 (1996), 2073-2080.

[23] M. Solomyak, On the spectrum of the Laplacian on regular metric trees, Special section on quantum graphs, Waves Random Media 14, 1 (2004), 155-171.

[24] A. WeBER, Analysis of the physical Laplacian and the heat flow on a locally finite graph, J. Math. Anal. Appl. 370, 1 (2010), 146-158.

[25] R. K. Wojciechowski, Stochastic completeness of graphs, Thesis (Ph.D.), City University of New York. 2008. 87 pp., ProQuest LLC, arXiv:0712.1570v2.

[26] R. K. WoJcIEchOWSKI, Stochastically incomplete manifolds and graphs, Boundaries and Spectral Theory, Progress in Probability, 2011, Birkhäuser. 